\title{
On multivariate higher order Lyapunov-type inequalities
}

Tieguo Ji* and Jie Fan

"Correspondence:
rosemary1976@163.com
College of Science, Hebei University
of Engineering, Handan, 056038,
P.R. China

"Correspondence: of Engineering, Handan, 056038

\begin{abstract}
In this paper, by using the best Sobolev constant method, we obtain some new Lyapunov-type inequalities for a class of even-order partial differential equations; the results of this paper are new which generalize and improve some early results in the literature.
\end{abstract}

Keywords: Lyapunov-type inequality; even-order partial differential equations; Sobolev constant

\section{Introduction}

It is well known that the Lyapunov inequality for the second-order linear differential equation

$$
x^{\prime \prime}(t)+q(t) x(t)=0
$$

states that if $q \in C[a, b], x(t)$ is a nonzero solution of (1) such that $x(a)=x(b)=0$, then the following inequality holds:

$$
\int_{a}^{b}|q(t)| d t>\frac{4}{b-a}
$$

and the constant 4 is sharp.

There have been many proofs and generalizations as well as improvements on this inequality. For example, the authors in [1-3] generalized the Lyapunov-type inequality to the partial differential equations or systems.

First let us recall some background and notations which are introduced in [1, 2].

Let $A$ be a spherical shell $\subseteq \mathbb{R}^{N}$ for $N>1$, i.e. $A=B\left(0, R_{2}\right)-\overline{B\left(0, R_{1}\right)}$ for $0<R_{1}<R_{2}$, where $B(0, R)=\left\{x \in \mathbb{R}^{N}:\|x\|<R\right\}$ for $R>0$ and $\|\cdot\|$ is the Euclidean norm. Denote $S^{N-1}=\{x \in$ $\left.\mathbb{R}^{N}:\|x\|=1\right\}$, the unit sphere in $\mathbb{R}^{N}$ with surface area

$$
\omega_{N}=\frac{2 \pi^{\frac{N}{2}}}{\Gamma\left(\frac{N}{2}\right)}, \quad \text { i.e. } \int_{S^{N-1}} d \omega=\frac{2 \pi^{\frac{N}{2}}}{\Gamma\left(\frac{N}{2}\right)}
$$

where $\Gamma(\cdot)$ is the gamma function. Then every $x \in \mathbb{R}^{N}-\{0\}$ has a unique representation of the form $x=r \omega$, where $r=\|x\|>0$ and $\omega=\frac{x}{r} \in S^{N-1}$. Therefore, for any $f \in C(\bar{A})$, we 
have

$$
\int_{A} f(x) d x=\int_{S^{N-1}}\left(\int_{R_{1}}^{R_{2}} f(r \omega) r^{N-1} d r\right) d \omega .
$$

In [1], Aktaş obtained the following results.

Theorem A If $\in C^{2 n}(\bar{A})$ is a nonzero solution of the following even-order partial differential equation:

$$
\frac{\partial^{2 n} f(x)}{\partial r^{2 n}}+q(x) f(x)=0, \quad x \in A,
$$

where $n \in \mathbb{N}$ and $q \in C(\bar{A})$, with the boundary conditions

$$
\frac{\partial^{2 i} f}{\partial r^{2 i}}\left(\partial B\left(0, R_{1}\right)\right)=\frac{\partial^{2 i} f}{\partial r^{2 i}}\left(\partial B\left(0, R_{2}\right)\right)=0, \quad i=0,1,2, \ldots, n-1,
$$

then the following inequality holds:

$$
\int_{A}|q(x)| d x>\frac{2^{3 n-1}}{\left(R_{2}-R_{1}\right)^{2 n-1}} \frac{2 \pi^{\frac{N}{2}}}{\Gamma\left(\frac{N}{2}\right)} R_{1}^{N-1}
$$

Theorem B If $\in C^{2 n}(\bar{A})$ is a nonzero solution of (4) with the boundary conditions

$$
\frac{\partial^{i} f}{\partial r^{i}}\left(\partial B\left(0, R_{1}\right)\right)=\frac{\partial^{i} f}{\partial r^{i}}\left(\partial B\left(0, R_{2}\right)\right)=0, \quad i=0,1,2, \ldots, n-1,
$$

then the following inequality holds:

$$
\int_{A}|q(x)| d x>\frac{4^{2 n-1}(2 n-1)[(n-1) !]^{2}}{\left(R_{2}-R_{1}\right)^{2 n-1}} \frac{2 \pi^{\frac{N}{2}}}{\Gamma\left(\frac{N}{2}\right)} R_{1}^{N-1} .
$$

In this paper, we generalize Theorem A and Theorem B to a more general class of even order partial differential equations. Moreover, as we shall see by the end of this paper, Theorem 1 improves Theorem A significantly.

\section{Main results}

Let us consider the following even-order partial differential equation:

$$
\frac{\partial^{2 n} y(x)}{\partial r^{2 n}}+\sum_{k=0}^{n} p_{k}(x) \frac{\partial^{k} y(x)}{\partial r^{k}}=0
$$

where $y(x) \in C^{2 n}(\bar{A}), p_{k}(x) \in C(\bar{A}), k=0,1,2, \ldots, n$, and $x \in \mathbb{R}^{N}$.

The main results of this paper are the following theorems.

Theorem 1 If $y(x)$ is a nonzero solution of (9) satisfying boundary conditions (5), then the following inequality holds: 


$$
\begin{aligned}
1< & \sqrt{\frac{\left(2^{2 n}-1\right) \zeta(2 n)\left(R_{2}-R_{1}\right)^{2 n-1} \Gamma\left(\frac{N}{2}\right)}{2^{2 n} \pi^{2 n+\frac{N}{2}}} R_{1}^{N-1}}\left(\int_{A} p_{n}^{2}(x) d x\right)^{\frac{1}{2}} \\
& +\sum_{k=0}^{n-1} \frac{\left(R_{2}-R_{1}\right)^{2 n-k-1} \Gamma\left(\frac{N}{2}\right)}{(2 \pi)^{2 n-k} R_{1}^{N-1} \pi^{\frac{N}{2}}} \sqrt{\left(2^{2 n}-1\right)\left(2^{2(n-k)}-1\right) \zeta(2 n) \zeta(2(n-k))} \\
& \times \int_{A}\left|p_{k}(x)\right| d x,
\end{aligned}
$$

where $\zeta(s)=\sum_{n=1}^{+\infty} \frac{1}{n^{s}}$ is the Riemann zeta function.

Theorem 2 If $y(x)$ is a nonzero solution of (9) satisfying boundary conditions (7), then the following inequality holds:

$$
\begin{aligned}
& 1<\frac{1}{(n-1) ! 2^{2 n-1}} \sqrt{\frac{\left(R_{2}-R_{1}\right)^{2 n-1} \Gamma\left(\frac{N}{2}\right)}{(2 n-1) R_{1}^{N-1} 2 \pi^{\frac{N}{2}}}}\left(\int_{A} p_{n}^{2}(x) d x\right)^{\frac{1}{2}} \\
& +\sum_{k=0}^{n-1} \frac{\left(R_{2}-R_{1}\right)^{2 n-k-1} \Gamma\left(\frac{N}{2}\right)}{\sqrt{(2 n-1)(2 n-2 k-1)(n-1) !(n-k-1) ! 4^{2 n-k-1} R_{1}^{N-1} 2 \pi^{\frac{N}{2}}}} \int_{A}\left|p_{k}(x)\right| d x .
\end{aligned}
$$

\section{Proofs of theorems}

For the proofs of Theorem 1 and Theorem 2, let us consider first the following ordinary even-order linear ordinary differential equation:

$$
x^{(2 n)}(t)+\sum_{k=0}^{n} p_{k}(t) x^{(k)}(t)=0
$$

where $p_{k}(t) \in C[a, b], k=0,1,2, \ldots, n$.

Proposition 3 If (12) has a nonzero solution $x(t)$ satisfying the following boundary value conditions:

$$
x^{(2 i)}(a)=x^{(2 i)}(b)=0, \quad i=0,1,2, \ldots, n-1,
$$

then the following inequality holds:

$$
\begin{aligned}
1< & \sqrt{\frac{\left(2^{2 n}-1\right) \zeta(2 n)(b-a)^{2 n-1}}{2^{2 n-1} \pi^{2 n}}\left(\int_{a}^{b} p_{n}^{2}(t) d t\right)^{\frac{1}{2}}} \\
& +\sum_{k=0}^{n-1} \frac{(b-a)^{2 n-k-1} \sqrt{\left(2^{2 n}-1\right)\left(2^{2 n-2 k}-1\right) \zeta(2 n) \zeta(2(n-k))}}{2^{2 n-k-1} \pi^{2 n-k}} \int_{a}^{b}\left|p_{k}(t)\right| d t
\end{aligned}
$$

where $\zeta(s)$ is the Riemann zeta function: $\zeta(s)=\sum_{k=1}^{+\infty} \frac{1}{k^{s}}, s>1$.

Proposition 4 If (12) has a nonzero solution $x(t)$ satisfying the following boundary value conditions:

$$
x^{(i)}(a)=x^{(i)}(b)=0, \quad i=0,1,2, \ldots, n-1,
$$


then we have the following inequality:

$$
\begin{aligned}
1< & \frac{1}{(n-1) ! 2^{2 n-1}} \sqrt{\frac{(b-a)^{2 n-1}}{(2 n-1)}}\left(\int_{a}^{b} p_{n}^{2}(t) d t\right)^{\frac{1}{2}} \\
& +\sum_{k=0}^{n-1} \frac{(b-a)^{2 n-k-1}}{(n-1) !(n-k-1) ! 4^{2 n-k-1} \sqrt{(2 n-1)(2 n-2 k-1)}} \int_{a}^{b}\left|p_{k}(t)\right| d t .
\end{aligned}
$$

In order to prove the above propositions, we need the following lemmas.

Lemma 5 ([4, Proposition 2.1]) Let $M \in \mathbb{N}$ and

$$
H_{C}=\left\{u \mid u^{(M)} \in L^{2}(a, b), u^{(2 k)}(a)=u^{(2 k)}(b)=0,0 \leq k \leq[(M-1) / 2]\right\} .
$$

Then there exists a positive constant $C$ such that, for any $u \in H_{C}$, the Sobolev inequality

$$
\left(\sup _{a \leq t \leq b}|u(t)|\right)^{2} \leq C \int_{a}^{b}\left|u^{(M)}(t)\right|^{2} d t
$$

holds. Moreover, the best constant $C=C(M)$ is as follows:

$$
C(M)=\frac{\left(2^{2 M}-1\right) \zeta(2 M)(b-a)^{2 M-1}}{2^{2 M-1} \pi^{2 M}} .
$$

Lemma 6 ([5, Theorem 1.2 and Corollary 1.3]) Let $M \in \mathbb{N}$ and

$$
H_{D}=\left\{u \mid u^{(M)} \in L^{2}(a, b), u^{(k)}(a)=u^{(k)}(b)=0,0 \leq k \leq M-1\right\} .
$$

Then there exists a positive constant $D$ such that for any $u \in H_{D}$, the Sobolev inequality

$$
\left(\sup _{a \leq t \leq b}|u(t)|\right)^{2} \leq D \int_{a}^{b}\left|u^{(M)}(t)\right|^{2} d t
$$

holds. Moreover, the best constant $D=D(M)$ is as follows:

$$
D(M)=\frac{(b-a)^{2 M-1}}{(2 M-1)[(M-1) !]^{2} 4^{2 M-1}} .
$$

We give the first seven values of $\zeta(2 n), C(n)$, and $D(n)$ in Table 1 .

Since the proof of Proposition 4 is similar to that of Proposition 3, we give only the proof of Proposition 3 below.

Table 1 The first seven values of $\zeta(2 n), C(n)$ and $D(n)$

\begin{tabular}{llllllll}
\hline $\boldsymbol{n}$ & $\mathbf{1}$ & $\mathbf{2}$ & $\mathbf{3}$ & $\mathbf{4}$ & $\mathbf{5}$ & $\mathbf{6}$ & $\mathbf{7}$ \\
\hline$\zeta(2 n)$ & $\frac{\pi^{2}}{6}$ & $\frac{\pi^{4}}{90}$ & $\frac{\pi^{6}}{945}$ & $\frac{\pi^{8}}{9,450}$ & $\frac{\pi^{10}}{93,555}$ & $\frac{691 \pi^{12}}{638,512,875}$ & $\frac{2 \pi^{14}}{18,23,225}$ \\
$C(n)$ & $\frac{b-a}{4}$ & $\frac{(b-a)^{3}}{48}$ & $\frac{(b-a)^{5}}{480}$ & $\frac{17(b-a)^{7}}{80,640}$ & $\frac{31(b-a)^{9}}{1,451,520}$ & $\frac{691(b-a)^{11}}{9,123,840}$ & $\frac{\left(2^{14}-1\right)(b-a)^{13}}{2^{13} \times 18,243,225}$ \\
$D(n)$ & $\frac{b-a}{4}$ & $\frac{(b-a)^{3}}{192}$ & $\frac{(b-a)^{5}}{20,480}$ & $\frac{(b-a)^{7}}{4,128,768}$ & $\frac{(b-a)^{9}}{1,358,954,496}$ & $\frac{(b-a)^{11}}{664,377,753,600}$ & $\frac{(b-a)^{13}}{13(6 !)^{2} 4^{13}}$ \\
\hline
\end{tabular}


Proof of Proposition 3 Multiplying both sides of (12) by $x(t)$ and integrating from $a$ to $b$ by parts and using the boundary value condition (13), we can obtain

$$
\int_{a}^{b} x^{(2 n)}(t) x(t) d t=(-1)^{n} \int_{a}^{b}\left(x^{(n)}(t)\right)^{2} d t=-\sum_{k=0}^{n} \int_{a}^{b} p_{k}(t) x^{(k)}(t) x(t) d t
$$

This yields

$$
\begin{aligned}
\int_{a}^{b}\left(x^{(n)}(t)\right)^{2} d t & \leq \sum_{k=0}^{n} \int_{a}^{b}\left|p_{k}(t)\right|\left|x^{(k)}(t) x(t)\right| d t \\
& =\int_{a}^{b}\left|p_{n}(t)\right|\left|x^{(n)}(t) x(t)\right| d t+\sum_{k=0}^{n-1} \int_{a}^{b}\left|p_{k}(t)\right|\left|x^{(k)}(t) x(t)\right| d t .
\end{aligned}
$$

Now, by using Lemma 5 , we get for any $t \in[a, b], k=1,2, \ldots, n-1$,

$$
|x(t)| \leq \sqrt{C(n)}\left(\int_{a}^{b}\left(x^{(n)}(t)\right)^{2} d t\right)^{\frac{1}{2}}
$$

and

$$
\left|x^{(k)}(t)\right| \leq \sqrt{C(n-k)}\left(\int_{a}^{b}\left(x^{(n)}(t)\right)^{2} d t\right)^{\frac{1}{2}} .
$$

Substituting (19) and (20) into (18), we obtain

$$
\begin{aligned}
\int_{a}^{b}\left(x^{(n)}(t)\right)^{2} d t \leq & \sqrt{C(n)} \int_{a}^{b}\left|p_{n}(t)\right|\left|x^{(n)}(t)\right| d t\left(\int_{a}^{b}\left(x^{(n)}(t)\right)^{2} d t\right)^{\frac{1}{2}} \\
& +\sum_{k=0}^{n-1} \sqrt{C(n) C(n-k)} \int_{a}^{b}\left|p_{k}(t)\right| d t \int_{a}^{b}\left(x^{(n)}(t)\right)^{2} d t
\end{aligned}
$$

Now by applying Hölder's inequality, we get

$$
\int_{a}^{b}\left|p_{n}(t) x^{(n)}(t)\right| d t \leq\left(\int_{a}^{b} p_{n}^{2}(t) d t\right)^{\frac{1}{2}}\left(\int_{a}^{b}\left(x^{(n)}(t)\right)^{2} d t\right)^{\frac{1}{2}}
$$

Substituting (22) into (21) and by using the fact that $x(t)$ is not a constant function, we obtain the following strict inequality:

$$
\begin{aligned}
\int_{a}^{b}\left(x^{(n)}(t)\right)^{2} d t< & \sqrt{C(n)}\left(\int_{a}^{b} p_{n}^{2}(t) d t\right)^{\frac{1}{2}} \int_{a}^{b}\left(x^{(n)}(t)\right)^{2} d t \\
& +\sum_{k=0}^{n-1} \sqrt{C(n) C(n-k)} \int_{a}^{b}\left|p_{k}(t)\right| d t \int_{a}^{b}\left(x^{(n)}(t)\right)^{2} d t .
\end{aligned}
$$


Dividing both sides of (23) by $\int_{a}^{b}\left(x^{(n)}(t)\right)^{2} d t$, which can be proved to be positive by using the boundary value condition (13) and the assumption that $x(t) \not \equiv 0$, we obtain

$$
1<\sqrt{C(n)}\left(\int_{a}^{b} p_{n}^{2}(t) d t\right)^{\frac{1}{2}}+\sum_{k=0}^{n-1} \sqrt{C(n) C(n-k)} \int_{a}^{b}\left|p_{k}(t)\right| d t .
$$

This is equivalent to (14). Thus we finished the proof of Proposition 3.

Lemma 7 For any $f \in C(A)$, we have

$$
\int_{A}|f(x)| d x \geq \frac{R_{1}^{N-1} 2 \pi^{\frac{N}{2}}}{\Gamma\left(\frac{N}{2}\right)} \int_{R_{1}}^{R_{2}}|f(r \omega)| d r
$$

Proof Similar to the proofs given in [1] and [2], we have

$$
\int_{R_{1}}^{R_{2}}|f(r \omega)| d r=\int_{R_{1}}^{R_{2}} r^{1-N} r^{N-1}|f(r \omega)| d r \leq\left(\int_{R_{1}}^{R_{2}} r^{N-1}|f(r \omega)| d r\right) R_{1}^{1-N}
$$

which implies that

$$
\begin{aligned}
\int_{A}|f(x)| d x & =\int_{S^{N-1}}\left(\int_{R_{1}}^{R_{2}} r^{N-1}|f(r \omega)| d r\right) d \omega \\
& \geq \int_{S^{N-1}}\left(R_{1}^{N-1} \int_{R_{1}}^{R_{2}}|f(r \omega)| d r\right) d \omega \\
& =\left(\int_{R_{1}}^{R_{2}}|f(r \omega)| d r\right) \frac{R_{1}^{N-1} 2 \pi^{\frac{N}{2}}}{\Gamma\left(\frac{N}{2}\right)} .
\end{aligned}
$$

Proof of Theorem 1 It follows from (14) and Lemma 7 that for any fixed $\omega \in S^{N-1}$, we have

$$
\begin{aligned}
1< & \sqrt{\frac{\left(2^{2 n}-1\right) \zeta(2 n)\left(R_{2}-R_{1}\right)^{2 n-1}}{2^{2 n-1} \pi^{2 n}}\left(\int_{R_{1}}^{R_{2}} p_{n}^{2}(r \omega) d r\right)^{\frac{1}{2}}} \\
& +\sum_{k=0}^{n-1} \frac{\left(R_{2}-R_{1}\right)^{2 n-k-1} \sqrt{\left(2^{2 n}-1\right)\left(2^{2 n-2 k}-1\right) \zeta(2 n) \zeta(2(n-k))}}{2^{2 n-k-1} \pi^{2 n-k}} \int_{a}^{b}\left|p_{k}(r \omega)\right| d r \\
\leq & \sqrt{\frac{\left(2^{2 n}-1\right) \zeta(2 n)\left(R_{2}-R_{1}\right)^{2 n-1} \Gamma\left(\frac{N}{2}\right)}{2^{2 n} \pi^{2 n+\frac{N}{2}} R_{1}^{N-1}}\left(\int_{A} p_{n}^{2}(x) d x\right)^{\frac{1}{2}}} \\
& +\sum_{k=0}^{n-1} \frac{\left(R_{2}-R_{1}\right)^{2 n-k-1} \Gamma\left(\frac{N}{2}\right)}{(2 \pi)^{2 n-k} R_{1}^{N-1} \pi^{\frac{N}{2}}} \sqrt{\left(2^{2 n}-1\right)\left(2^{2(n-k)}-1\right) \zeta(2 n) \zeta(2(n-k))} \int_{A}\left|p_{k}(x)\right| d x,
\end{aligned}
$$

which is (10). This finishes the proof of Theorem 1.

The proof of Theorem 2 is similar to that of Theorem 1, so we omit it for simplicity. Let us compare Theorem 1 and Theorem 2 with Theorem A and Theorem B. It is evident that Theorem 2 is a natural generalization of Theorem B. If we let $p_{n}(x)=p_{n-1}(x)=\cdots=$ 
Table 2 The first eight values of $\delta_{n}$

\begin{tabular}{lllllllll}
\hline $\boldsymbol{n}$ & $\mathbf{1}$ & $\mathbf{2}$ & $\mathbf{3}$ & $\mathbf{4}$ & $\mathbf{5}$ & $\mathbf{6}$ & $\mathbf{7}$ & $\mathbf{8}$ \\
\hline$\delta_{n}$ & 1 & 1.50 & 1.42 & 2.32 & 2.86 & 3.53 & 4.35 & 5.37 \\
\hline
\end{tabular}

$p_{1}(x) \equiv 0, p_{0}(x)=q(x), \forall x \in A$, then (10) reduces to the following inequality:

$$
\int_{A}|q(x)| d x>\frac{2^{2 n-1} \pi^{2 n}}{\left(2^{2 n}-1\right) \zeta(2 n)\left(R_{2}-R_{1}\right)^{2 n-1}} \frac{2 \pi^{\frac{N}{2}}}{\Gamma\left(\frac{N}{2}\right)} R_{1}^{N-1} .
$$

Let us compare the right sides of inequalities (6) and (25): if we denote $\delta_{n}=\frac{2^{2 n-1} \pi^{2 n}}{\left(2^{2 n}-1\right) \zeta(2 n) 2^{3 n-1}}$, then we have

$$
\delta_{n}=\frac{\pi^{2 n}}{2^{n}\left(2^{2 n}-1\right) \zeta(2 n)}>\frac{\pi^{2 n}}{2^{3 n} \zeta(2 n)}=\left(\frac{\pi^{2}}{8}\right)^{n} \frac{1}{\zeta(2 n)} \rightarrow \infty, \quad \text { as } n \rightarrow \infty,
$$

since $\zeta(2 n) \rightarrow 1$ as $n \rightarrow \infty$. Table 2 gives the first eight values of $\delta_{n}$.

From Table 2 we see that $\delta_{n}$ increases very quickly, so Theorem 1 improves Theorem A significantly even in the special case of (4).

\section{Competing interests}

The authors declare that they have no competing interests.

\section{Authors' contributions}

All authors contributed equally to the writing of this paper. All authors read and approved the final manuscript.

\section{Acknowledgements}

The authors thank the anonymous referees for their valuable suggestions and comments on the original manuscript.

Received: 30 September 2014 Accepted: 1 December 2014 Published: 12 Dec 2014

\section{References}

1. Aktas, MF: On the multivariate Lyapunov inequalities. Appl. Math. Comput. 232, 784-786 (2014)

2. Anastassiou, GA: Multivariate Lyapunov inequalities. Appl. Math. Lett. 24, 2167-2171 (2011)

3. Canada, A, Montero, JA, Villegas, S: Laypunov inequalities for partial differential equations. J. Funct. Anal. 237, 176-193 (2006)

4. Watanabe, K, Yamagishi, H, Kametaka, Y: Riemann zeta function and Lyapunov-type inequalities for certain higher order differential equations. Appl. Math. Comput. 218, 3950-3953 (2011)

5. Watanabe, K, Kametaka, Y, Yamagishi, H, Nagai, A, Takemura, K: The best constant of Sobolev inequality corresponding to clamped boundary value problem. Bound. Value Probl. 2011, Article ID 875057 (2011). doi:10.1155/2011/875057

10.1186/1029-242X-2014-503

Cite this article as: Ji and Fan: On multivariate higher order Lyapunov-type inequalities. Journal of Inequalities and Applications 2014, 2014:503

\section{Submit your manuscript to a SpringerOpen ${ }^{\ominus}$ journal and benefit from:}

- Convenient online submission

- Rigorous peer review

- Immediate publication on acceptance

- Open access: articles freely available online

- High visibility within the field

- Retaining the copyright to your article 